1794-9831

E-ISSN 2322-7028

Vol. 15 No. 1

Ene - Jun 2018

Cúcuta, Colombia

Recibido:

5 Mayo

2017

Aprobado:

31 Octubre

2017

Autor de correspondencia

$\triangle$ Psicóloga. Magíster en Salud Colectiva - Universidad de Antioquia Psicóloga. Liga Antioqueña de $\mathrm{He}$ mofílicos. Orcid 0000-0001-98546113. Correo: yuryjad@hotmail com. Medellín, Colombia.

\section{Significados de ser portadoras de hemofilia $\dagger$}

\author{
Significance of being a hemophilia carrier
}

Significado de ser portadora de hemofilia

\author{
Yuri Andrea Arango Bernal*
}

\section{Resumen}

Objetivo: Analizar los significados que construyen las madres de personas en condición de hemofilia, sobre ser portadoras de la enfermedad. Materiales y Métodos: Estudio cualitativo con enfoque del interaccionismo simbólico que, a través de una etnografía particularista y el uso de entrevistas semiestructuradas, observaciones y revisión documental, rescató el punto de vista de 17 madres pertenecientes a la Liga Antioqueña de Hemofílicos que participaron de manera voluntaria y residen en diferentes municipios del departamento de Antioquia. Resultados: Las participantes se reconocen a sí mismas como seres potenciales, es decir, no sólo como trasmisoras o cuidadoras de la enfermedad de sus hijos, sino como sujetos cognoscentes de su realidad dispuestas a reflexionar sobre sus aprendizajes e incorporarlos a favor de la relación consigo mismas y con los demás. Los significados más relevantes son: empezar a vivir con hemofilia, asumir la enfermedad, la familia, el cuidado y la crianza, la relación con los servicios de salud, caminando con algo que no se puede desprender y darse cuenta. Conclusiones: La salud colectiva es la posibilidad de tener un acercamiento más comprensivo al proceso salud - enfermedad - atención de los colectivos humanos, tomando en cuenta las condiciones económicas, sociales y culturales en las que estos se inscriben. La pregunta por los significados de estas madres develó el tejido de lo individual y lo colectivo, como un asunto que trasciende el plano biológico de la enfermedad y da cuenta de la construcción social en la que confluyen prácticas, saberes, imaginarios y sentimientos.

Palabras Clave: Cuidadores, enfermedad crónica, hemofilia A, madres

\section{Abstract}

Objective: To analyze the significance built by mothers of people with hemophilia about being a carrier of this disease. Materials and methods: Qualitative study, focused on the symbolic interaction that, through a particular ethnography and the use of semi structured interviews, observations and documental reviews, rescued the point of view of 17 mothers that made part of the Antioquia League of Hemophiliacs, and voluntarily participated and reside in different municipalities of the Antioquia department. Results: The participants recognize themselves as potential people, meaning, not only as transmitters or caregivers of the disease of their children, but also as connoisseurs of their reality, willing to reflect about their knowledge and incorporate them in favor of the relationship with themselves and everybody else. The most relevant significances are: start living with hemophilia, assume the disease, the family, the care and the parenting, the relation with health services, and walking with something they cannot take off and realizing it. Conclusions: The collective health is the possibility of having a close and more comprehensive encounter with the process of healthdisease-care in human communities, considering the economic, social and cultural conditions in which these are involved. The question about the significance of these mothers unveiled the bond of

$\dagger$ Este Articulo es producto de la investigación "Significados de ser portadora de hemofilia: el caso de un grupo de madres pertenecientes a la Liga Antioqueña de Hemofílicos”. Requisito para optar al título de Magíster en Salud Colectiva 2013-2015. Adscrita al Grupo de Investigación Políticas Sociales y Servicios de Salud - Universidad de Antioquia. Trabajo realizado en la Facultad de Enfermería U de A.

\section{Para citar este artículo/ To reference this article/ Para citar este artigo/}

Arango Bernal YA. Significados de ser portadoras de hemofilia. Rev. cienc. cuidad. 2018;15(1): 18-33

Este es un artículo bajo la licencia CC BY (https://creativecommons.org/licenses/by/4.0/) @ () 
notes the social construction in which practices, knowledge and feelings combine.

ISSN-PRINT

$1794-9831$

E-ISSN 2322-7028

Vol. 15 No. 1

Keywords: Caregivers, chronic disease, hemophilia A, mothers

\section{Resumo}

Objetivo: Analisar os significados que constroem as mães de pessoas em condição de hemofilia, sobre ser portadoras da doença. Materiais e Métodos: Estudo qualitativo com enfoque do interacionismo simbólico que, através de uma etnografia particularista e o uso de entrevistas semiestruturadas, observações e revisão documental, resgatou o ponto de vista de 17 mães pertencentes à Liga Antioqueña de Hemofílicos que participaram de maneira voluntaria e residem em diferentes municípios do estado de Antioquia, Colômbia. Resultados: As participantes se reconhecem a si mesmas como seres potenciais, é dizer, não só como transmissoras ou cuidadoras da doença de seus filhos, senão como sujeitos cognoscentes de sua realidade e dispostas a refletir sobre suas aprendizagens e incorporá-las a favor da relação consigo mesmas e com os outros. Os significados mais relevantes são: empezar a viver com hemofilia, assumir a doença, a família, o cuidado e a cria, a relação com os serviços de saúde, caminhando com algo que não se pode desprender e dar-se conta. Conclusões: A saúde coletiva é a possibilidade de ter uma abordagem mais compreensiva ao processo saúde - doença - atendimento dos coletivos humanos, tomando em conta as condições econômicas, sociais e culturais nas quais estes se inscrevem. A pergunta pelos significados destas mães mostrou o tecido do individual e do coletivo, como um assunto que transcende o plano biológico da doença e da conta da construção social na que confluem práticas, saberes, imaginários e sentimentos.

Palavras-chave: Cuidadores, doença crônica, hemofilia A, mães

\section{Introducción}

Las hemofilias A y B son los principales trastornos de la coagulación, de carácter hereditario, ligados al cromosoma sexual $\mathrm{X}$, que se presentan debido a mutaciones en los genes del factor VIII (hemofilia A, HA) y factor IX (hemofilia B, HB), ocasionando una disminución o deficiencia funcional de estas proteínas en plasma. Sus frecuencias son de 1 en 5,000 y 1 en 30,000 varones recién nacidos vivos, respectivamente. Estos genes se localizan en el cromosoma sexual X, razón por la cual su patrón de herencia es recesivo ligado a este, lo que afecta casi exclusivamente a varones, teniendo las mujeres portadoras un riesgo del $50 \%$ de heredarlo a sus hijos (1).

Los síntomas de la enfermedad no se presentan de manera agravada en las mujeres porque la $\mathrm{X}$ sana suple la deficiencia de la X que porta la información genética de la enfermedad, contrario a lo que sucede en los varones, en quienes su cromosoma $\mathrm{X}$ presenta la mutación de la hemofilia y no tiene como suplir la deficiencia, pues su otro cromosoma es Y.

Algunas mujeres portadoras (2) pueden tener niveles de factor de coagulación dentro de los rangos de la hemofilia -principalmente en categoría leveEn casos poco comunes pueden estar dentro de los rangos moderados o severos, debido a una lionización extrema del cromosoma $\mathrm{X}$.

Las portadoras con niveles de factor de coagulación dentro de los rangos de la hemofilia pueden presentar manifestaciones hemorrágicas correspondientes al grado de deficiencia de factor de coagulación, particularmente durante traumatismos y cirugías (2). Desde esta perspectiva, las portadoras deberían clasificarse como personas con hemofilia y recibir el tratamiento de acuerdo con la gravedad del trastorno.

En este sentido, en países más avanzados en relación con el tratamiento de la enfermedad, a las portadoras se les presta orientación genética a través de pruebas de laboratorio, asesoría familiar y acompañamiento en las opciones reproductivas; es decir, las opciones de los padres de un niño con hemofilia recién diagnosticada son las mismas que para otras portadoras potenciales antes de la concepción: contracepción, transferencia intratubaria de gametos (GIFT), aborto después de una muestra de la membrana coriónica, FIV/selección de sexo y adopción (3). 
ISSN-PRINT

1794-9831

E-ISSN 2322-7028

Vol. 15 No. 1

Ene - Jun 2018

Cúcuta, Colombia
En Colombia (4), las formas de acceso al tratamiento de la enfermedad y a los programas de acompañamiento presentan diferencias, puesto que se establecen unas acciones para quien padece los síntomas y otras para quien porta la enfermedad. Las portadoras de hemofilia no cuentan con los exámenes de laboratorio suficientes para un diagnóstico certero; ellas saben que son portadoras a partir del diagnóstico de sus hijos, debido a que no hay orientación genética suficiente y las redes de apoyo se orientan prioritariamente hacia los pacientes y no hacia las mujeres portadoras, quienes, en su mayoría, no presentan síntomas ni secuelas importantes propias de la enfermedad.

Además, se observa que las madres portadoras son las principales cuidadoras de la enfermedad de sus hijos, son quienes hacen el esfuerzo por aprender del tema, están al frente de las situaciones de crisis, reclaman los medicamentos, realizan los trámites en la institución de salud y facilitan a sus hijos las estrategias, herramientas y acciones para que puedan crecer con mejores opciones de tratamiento y de vida.

Los avances en la ciencia y la tecnología permiten identificar que el interés primordial en cuanto a la hemofilia se ha centrado en el tratamiento. Por una parte, se cuenta con un mejor manejo de la enfermedad, ya que el conocimiento de la naturaleza del defecto genético (5) permite reducir considerablemente los riesgos de complicaciones clínicas asociadas a ésta. En Colombia también se han iniciado investigaciones que apuntan al reconocimiento del diagnóstico molecular para enriquecer las alternativas de tratamiento, encaminadas a brindar la posibilidad preventiva mediante estrategias diagnósticas de detección de portadoras, con el fin de permitir el establecimiento de un diagnóstico precoz, como el prenatal o la pre-implantación genética o estudio del ADN de embriones humanos para seleccionar los que cumplen determinadas características y/o eliminar los que portan algún tipo de defecto congénito (6).

La asesoría genética en hemofilia (7) constituye una parte importante del cuidado, ya sea en países que se encuentran a la vanguardia en diagnóstico y tratamiento o en los que cuentan con pocas facilidades para ello, lo cual indica que, con ciertos conocimientos, es posible ayudar a personas con la enfermedad, a las portadoras y a sus familias, a tomar decisiones más acertadas e informadas con respecto a tener hijos cuando existe la posibilidad de que éstos nazcan con hemofilia.

Este panorama convoca a una reflexión sobre el tema de la hemofilia y las madres portadoras de la misma, desde el hecho de ser mujer hasta la relación con el proceso de salud enfermedad que las envuelve, puesto que, precisamente, son las mujeres quienes tienen un papel relevante en la familia, la sociedad y la cultura, centrado especialmente en el cuidado de los hijos y el acompañamiento para el uso de los servicios de salud.

Con este interés especial y a la luz de la salud colectiva, como una forma de favorecer el acercamiento a las inquietudes que se presentan en torno al tema, se forjó la motivación por las madres portadoras de hemofilia que, como menciona Neira (8), "este redimensionamiento del objeto de estudio plantea, a su vez, nuevos interrogantes en relación con la vinculación y las complejidades diversas que se enfrentan entre las manifestaciones individuales y el nivel colectivo. Si bien la manifestación individual es resultado de lo colectivo y lo colectivo se ve implicado por lo individual, ambos son cualitativamente diferentes".

En virtud de lo anterior, este trabajo se inscribe en la propuesta de la salud colectiva, desde la cual se tienen en cuenta los aspectos genéticos y los biológicos, se reconoce el valor de otras esferas como lo subjetivo, lo cotidiano, lo individual y lo social, en tanto procesos históricamente determinados.

En este sentido, la salud colectiva puede aportar de manera importante al desarrollo de nuevas propuestas específicas alrededor de enfermedades como la hemofilia, que, aunque son de baja incidencia, tienen gran impacto en todas las esferas de la persona y del Sistema de Seguridad Social en Salud. Así mismo, pensar en procesos de gestión social en salud más articulados, más humanizados y, por supuesto, que impacten en la calidad de vida de quienes conviven con la enfermedad como portadoras de la misma.

\section{Objetivos}

\section{Objetivo general}

Analizar los significados que construyen las madres de personas en condición de hemofilia, sobre ser portadoras de la enfermedad. 


\section{Objetivos específicos}

- Explorar las implicaciones personales que conlleva ser madre de una persona en condición de hemofilia.

- Reconocer los cambios que se dan en la vida cotidiana de las madres portadoras por el hecho de tener un hijo en condición de hemofilia.

- Identificar, desde las voces de las madres, la forma en que se relacionan con su entorno familiar y social.

\section{Materiales y Métodos}

Para el análisis de los significados se recurrió al método cualitativo apoyado en el enfoque del interaccionismo simbólico, el cual recupera la mirada sobre realidades subjetivas e intersubjetivas para comprender -desde la interioridad de los actores sociales- las lógicas de pensamiento que guían sus acciones (9) y los significados que las personas asignan al mundo que les rodea.

La modalidad que permitió el acercamiento a esta realidad fue la etnografía particularista, la cual permite moverse en grupos poblacionales pequeños (9). Así mismo, siendo la hemofilia una enfermedad de baja incidencia, los casos que se presentan en la población son pocos. Se realizaron entrevistas semiestructuradas a 17 mujeres portadoras que pertenecen a la Liga Antioqueña de Hemofílicos, quienes participaron voluntariamente. El muestreo se hizo pensando en las madres protagonistas que, desde la investigación cualitativa, son las que hablan desde sus propias experiencias y vivencias (10).

Al respecto, se tuvieron en cuenta los siguientes criterios de inclusión: madres que pertenecieran al grupo de apoyo de la Liga Antioqueña de Hemofílicos y quisieran participar de manera voluntaria, que tuvieran claro el diagnóstico de sus hijos -pues son las que ciertamente saben que son portadoras-, y que tuvieran el diagnóstico de sus hijos a partir de un año, ya que tal vez las de poco tiempo de reconocimiento del diagnóstico estén realizando un ajuste importante e indagar por ello podría resultar intrusivo y afectar su situación personal.

El número de entrevistas realizadas se derivó de la saturación de los datos (10), la cual se alcanza cuando hay redundancia en la información, esto es, cuando el investigador obtiene la misma información o similar, pues los informantes no indican algo diferente de lo ya dicho.

La mayoría de las participantes fueron mujeres en edad adulta, entre los 27 y 60 años -3 de ellas jóvenes entre los 14 y los 26 años, y 2 mayores de 60 años-. Se trata de una población diversa, característica que dio pistas para el análisis de la información. Diez de las participantes viven en el área Metropolitana de Antioquia y 7 fuera de ésta.

Para las entrevistas se implementó una guía diseñada en el marco de los objetivos, con el propósito de orientar los espacios de encuentro de acuerdo con el propósito del estudio y en aras de favorecer la reflexión desde las categorías de análisis. La guía constó de tres partes: objetivos, preguntas centrales y preguntas complementarias, las cuales permitieron profundizar en los temas sin desenfocarse del propósito principal.

Teniendo en cuenta que la observación participante, según Sandoval (11), es la principal herramienta de trabajo de la etnografía y se emplea para definir el problema de investigación con referencia a la vida cotidiana de las personas, se considera una estrategia flexible de apertura y cierre.

Para la observación se elaboró una guía basada en las directrices que sugieren Le Compte y Goetz (12), quienes plantean que las guías son marcos de referencia para que los investigadores se puedan centrar en los objetivos a estudiar, proporcionando focos de observación y registro, pero, debido al flujo interaccional de la realidad, es muy complejo pretender captar las cosas en su totalidad.

De esta forma, la observación participante se dio en cuatro momentos del trabajo y a cada una se le realizó diario de campo, con el propósito de documentar los aspectos más importantes relacionados con el objeto de estudio. Los espacios físicos fueron diversos y aportaron aspectos relevantes para el análisis de la información recolectada, en concordancia con las entrevistas y demás proceso transversales.

Otro aspecto que aportó al proceso de recolección de la información fue la revisión documental como aspecto transversal del estudio, actividad que 
ISSN-PRINT

1794-9831

E-ISSN 2322-7028

Vol. 15 No. 1

Ene - Jun 2018

Cúcuta, Colombia favoreció la búsqueda constante del tema central y las categorías de análisis o aquellas que emergieron en el transcurso del trabajo. Dicha revisión permitió concretar asuntos teóricos y tomar otras decisiones pertinentes. Además, se utilizaron notas de trabajo que ayudaron a vislumbrar nuevas ideas y a plasmar una postura creativa propia de la investigación cualitativa, poniéndose de presente la relación con las participantes y con todos los elementos de la investigación que se veían permeados por la vida cotidiana, por el desarrollo profesional y por la construcción académica.

El análisis se realizó en paralelo con la recolección de información, se elaboraron fichas de contenido que, como sugiere Galeano (13), favorecen el análisis e interpretación de la información en cuanto sirven de base para la construcción y afinación de categorías de análisis, posibilitan la triangulación de teorías, fuentes o técnicas de recolección de datos, además de diferenciar las percepciones y argumentos del investigador y las de los participantes, evidenciando vacíos.

Con el propósito de hacer un tratamiento adecuado de los datos, se efectuó una codificación abierta para empezar y, posteriormente, una codificación axial (14) hasta encontrar categorías interpretativas que dieran cuenta de una postura relacional desde las voces de las participantes como si tuvieran vida propia, y una construcción teórica más estructurada.

Las consideraciones éticas fueron un aspecto de reflexión constante desde la perspectiva de la investigación social. Al respecto, se retomó a Menéndez (15), quien plantea la importancia del punto de vista del actor. También se tuvieron en cuenta las consideraciones éticas, de acuerdo con las orientaciones internacionales de investigación biomédica (16) y la Resolución 008430 de 1993 del Ministerio de Salud de Colombia (17) con relación al respeto por la dignidad humana y los principios éticos de justicia, beneficencia no maleficencia, solidaridad y respeto por la autonomía. Así mismo, se contó con el aval del Comité de Ética de la Investigación de la Facultad de Enfermería de la Universidad de Antioquia (Acta No CEI-FE 2014- del 26 de VI de 2014).

\section{Resultados}

Analizar los significados supone apertura, relectura y disposición a explorar, es decir, los datos no llegan de facto, puesto que surgen en un proceso en donde prima lo cambiante en el sentido de que cada suceso o experiencia subjetiva que los refleja expresa una relación de acciones recíprocas a través de lo que se vive en simultáneo; no es la suma de testimonios.

Castro (18) afirma que la experiencia subjetiva depende tanto de la naturaleza de la estructura social como de la visión que los actores tienen de ella. La manera en que los individuos dan cuenta de la experiencia está estrechamente relacionada con sus condiciones objetivas de vida y con los patrones de sentido común que utilizan para explicar dichas condiciones. Los individuos aprecian su orden social dentro del contexto de ciertos patrones de sentido común que, junto con el orden social, se ven reflejados también en sus relatos.

Teniendo en cuenta estas consideraciones y que en el análisis de los significados primó la mirada subjetiva como fuente de conocimiento de la realidad de las participantes, éstos se presentan por medio de categorías que permiten analizarlos de manera particular y, a su vez, desde esferas más grandes con las que se quiere expresar la complejidad de los mismos. La siguiente gráfica ilustra las principales categorías que agrupan los significados que emergieron a la luz de los testimonios de las participantes: 


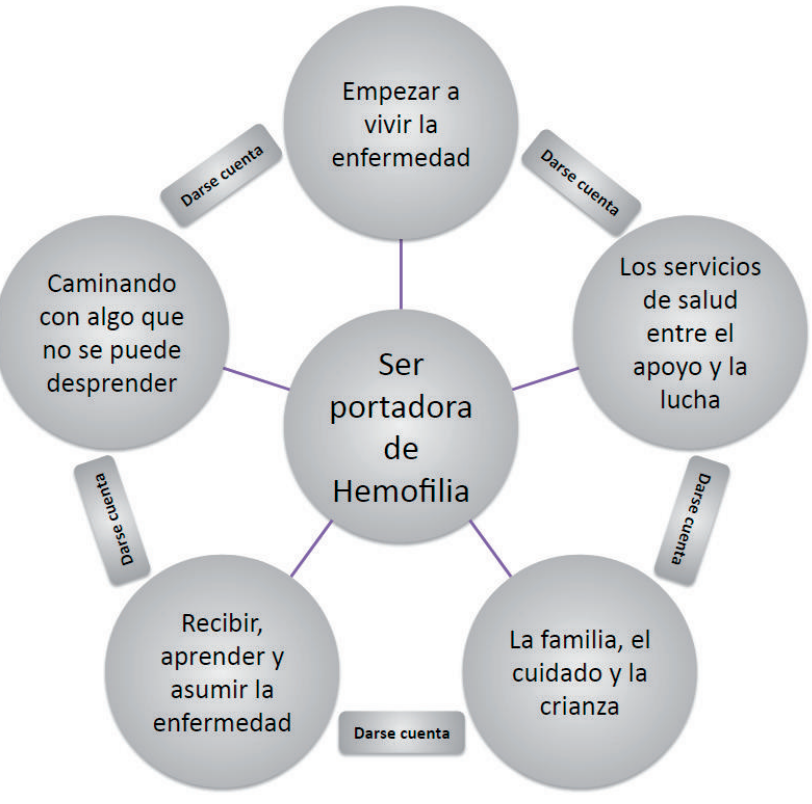

ISSN-PRINT

1794-9831

E-ISSN 2322-7028

Vol. 15 No. 1

Ene - Jun 2018

Cúcuta, Colombia

Figura 1: Categorías que representan los significados de ser portadoras de hemofilia.

Fuente: Proceso de análisis de la investigación Significados de ser portadoras de Hemofilia, el caso de un grupo de madres pertenecientes la Liga Antioqueña de Hemofílicos, 2015. Yuri Andrea Arango Bernal.

\section{Empezar a vivir con hemofilia: entre esperar la enfermedad y albergar la esperanza}

Vivir con hemofilia, para las madres portadoras -el binomio madre/hijo es atravesado por la experiencia de padecimiento y las características de la enfermedad (madre portadora del gen con hemofilia)- representa cambios que se ven afectados por el diagnóstico y, en la mayoría de los casos, es confirmación de algo que se espera y que a través del tiempo va cambiando. Para las madres, en un principio, el reconocimiento de la hemofilia se traduce en negaciones y aceptaciones a partir del acercamiento que se da en la relación con sus hijos y en el manejo de la enfermedad.

Todo lo anterior corresponde a un proceso de concientización en donde toma fuerza, para la madre, el carácter de cuidadora y de portadora (trasmisora) de la enfermedad. Es por ello que empezar a vivir con un hijo que padece la enfermedad se relaciona con varios aspectos que se enlazan: por una parte, se manifiestan los sentimientos de incertidumbre -si son portadoras o no- que tienen las madres con antecedentes familiares de la enfermedad y, por la otra, se presentan casos de mujeres que no sabían acerca de ésta y eso representa para ellas una noticia crucial y desestabilizadora.

Una de las madres portadoras de hemofilia cuenta que la enfermedad de su hijo se hizo evidente cuando empezó a notar algunos cambios en el cuerpo: Para ella, esos cambios no eran ajenos debido a que, cuando era niña, supo de algunos familiares que habían tenido hemofilia:

"Mi hijo nació muy hermoso, rojito, lindo, yo pensé que no tenía ninguna clase de cosa, [...] a los seis meses que empezó a voltearse en la cuna, se le pusieron los hombros inegros! Entonces, cuando llegó mi esposo, yo estaba llorando y le dije ;ay! el niño mío es hemofilico”. E3

Este es el caso de la mayoría de las participantes que intuían la enfermedad y que esperaban que no fuera su caso. Al considerar los testimonios de las participantes que ya reconocían la enfermedad, se destaca la importancia de la espera de ésta en su propia vida, a través de los padecimientos de sus hijos, lo que, unido a las situaciones vividas por sus familiares, les permite reconocer los sucesos que se pueden desatar a causa de la hemofilia. 
ISSN-PRINT

1794-9831

E-ISSN 2322-7028

Vol. 15 No. 1

Ene - Jun 2018

Cúcuta, Colombia
Esta experiencia anterior en la familia les permite reconocer algunas características que genera la sensación intuitiva del proceso y que se presentan, como simbolismo clave, mediante manifestaciones físicas en el cuerpo tales como morados, hinchazón y sangrados abundantes -en el caso de los accidentes donde se presentan heridas abiertas- todos ellos como principal señal de que su hijo será diagnosticado con hemofilia.

Las madres reconocen que sus hijos están presentando señales en su cuerpo que implican cambios en la realización de sus actividades cotidianas y su comportamiento, fenómeno que hace que ellas recreen en su pensamiento la posibilidad de tener un hijo en condición de hemofilia. Para otra de las participantes, quien vivió con tres familiares que padecieron la enfermedad, su decisión de tener un hijo y conformar un hogar prevaleció en el tiempo, pero en el momento en que llegó la enfermedad a su vida, se convirtió en un "dolor de cabeza" porque sabía lo que podía pasar: “... a los tres meses empezó mi dolor de cabeza porque empecé a notar que lloraba mucho, se estaba hinchando mucho en esta parte de las orejas, le dolía, lloraba mucho, entonces yo ya empecé a darme cuenta". E10

Dado que la enfermedad se hizo presente en la vida de sus familiares, su experiencia como hermanas, hijas u otro rol en la familia de una persona que padece hemofilia, generó que las madres comenzaran a construir los significados en relación con este padecimiento, y es a partir del diagnóstico de sus hijos que se concretó la experiencia como portadoras, es decir, tomó forma en su propia vida lo que es ser madre de una persona en condición de hemofilia. En otras palabras, descubrir la enfermedad de sus hijos es descubrirse ellas como portadoras de la misma. De esta forma, cuando sus hijos son diagnosticados con la enfermedad, ellas también son diagnosticadas, ya sea por medio de la realización de pruebas de laboratorio o por los antecedentes familiares de la enfermedad.

En cuanto al hecho de reconocerse como portadoras de hemofilia, las madres asumen una posición de cuidadoras primordiales que, en parte, se debe al hecho mismo de ser la mamá. A esto se suma que existe una cierta responsabilidad que las participantes reconocen más como un proceso que se trasfiere en la relación con los otros a través de las apreciaciones que les imparten, como ocurre en el caso de los profesionales de la salud y las ideas que les traspasan los familiares cercanos y lejanos, que en su mayoría están cargados de cierto prejuicio.

Sin embargo, este suceso es diferente entre las madres que tienen familiares que la padecen y aquellas que no tienen antecedentes de la enfermedad -para quienes el proceso es desconocido hasta que los hallazgos de laboratorio, ordenados por los médicos, llevan al diagnóstico-. Este es el caso de una participante que no tenía antecedentes de la enfermedad en su familia y refiere el momento de recibir la noticia:

"...fue súper duro porque, cuando nos dijeron que esa enfermedad la trataba un hematólogo, pues obviamente uno lo asocia con cáncer de sangre, sentía que se me cerraban las puertas". E17

Este testimonio refleja cómo ella se vio abrumada por la noticia debido a que no conocía sobre la enfermedad ni se sabía portadora de ella, lo que hace aún más difícil integrar esta realidad a su vida.

De igual forma, un aspecto que se resalta en este testimonio y en el de las demás participantes que no sabían acerca de la enfermedad, consiste en la importancia de la comunicación que los profesionales de la salud les dan en ese momento y la información que les imparten: por una parte, porque es el primer reconocimiento de la hemofilia y, por la otra, porque influye en gran medida en la forma como la perciben para afrontar el proceso de vivir con la enfermedad de su hijo. Una de ellas manifiesta, al respecto, que fue decisivo el momento en que el médico le dio la noticia del diagnóstico de su primer hijo, hace 28 años:

“... A mi me dio mucha tristeza. Yo, mejor dicho, se lo digo, como que se me derrumbó el mundo, porque a mí eso me parecía horrible, porque, como no había tanta información como ahora, eso le decían a uno así como a la carrera, dizque, ah, es que tiene una enfermedad en la sangre y cuidadito lo dejan caer, no lo vaya a dejar cortar, tiene que cuidarlo mucho, no lo puede dejar hacer educación fisica". E2

En ese momento, surgen pensamientos confusos por no entender lo que está pasando. En algunos casos se da la no aceptación como un mecanismo de control temporal sobre la situación, hasta que se puedan dar ajustes frente a la noticia, de modo que las madres logran asumir la situación de enfermedad de sus hijos en diferentes ritmos, con el fin de sobrellevarla. En algunos testimonios son notorios estos sentimientos: "Eso es como un baldado de agua fría; yo pensaba Dios mío, ahora ¿qué voy hacer? ¿por dónde voy empezar?, eso lo asusta a uno". E4 
"Yo me puse a llorar mucho, me desmayé porque yo no aceptaba lo que estaba pasando”. E3

Se observa la frustración de las madres en el momento, al no poder cambiar el diagnóstico ni controlar el curso de los acontecimientos, porque la hemofilia es una enfermedad incurable, lo que hace que la noticia se asuma con más incertidumbre por parte de ellas, percibiendo estos sentimientos como una reacción de afrontamiento para aceptar, en mayor medida, la condición de su hijo como una parte de su vida.

Es impreciso asegurar que todas las madres portadoras de hemofilia aceptan la condición prontamente, pero sí se puede evidenciar que, pese a las vicisitudes que genera la enfermedad, en ellas se expresa una fuerza interior que les permite afrontarla y asumirla.

Los sentimientos inicialmente son diversos: llanto, tristeza y conmoción, por un lado, por tener una idea de lo que puede suceder; por otro lado, el desconcierto de no querer aceptar esta situación. En ambos casos, descubrir la enfermedad y recibir el diagnóstico da inicio a una nueva vida con múltiples dificultades y luchas, es una circunstancia que impone a las madres un nuevo reto frente a la vida, la crianza y la dinámica de la familia.

\section{Asumir la enfermedad}

En todos los casos, para las madres es difícil asumir la enfermedad de sus hijos por diferentes motivos, entre los que predominan la sensación de lo incurable, el tiempo que los tendrán con vida, el trato que se les debe brindar y el darse cuenta de que ellas portan la enfermedad y deben aceptar la condición de portadoras.

En estas madres se puede apreciar cierta negación al hecho de reconocerse como portadoras de hemofilia, puesto que lo identifican como algo que les hacen saber o que desde afuera les reconocen, más que como un proceso interiorizado de manera natural hacia sí mismas. Las madres conciben el hecho de ser las portadoras de la enfermedad como algo no grato, inesperado, difícil de aceptar y hasta como asunto de suerte. Una de ellas se expresa al respecto:

“...siempre se ha escuchado de que, como es una enfermedad hereditaria, que es genética, pues se supone que las hermanas somos portadoras y en este caso de las tres mujeres, me tocó a mí ser la portadora”. E1

La expresión de la participante denota falta de información sobre la enfermedad, pues lo manifiesta como un asunto de suerte del que sus hermanas se libraron; quizás no tiene claro que todas sus hermanas son portadoras también y que el asunto es si ellas tuvieron o no hijos hombres que podrían estar sanos o con hemofilia.

Es característico, entre las madres, recibir información acerca de la enfermedad y hacer sus apreciaciones con respecto a la misma, aunque la información también las hace tomar posiciones diversas y en ocasiones contradictorias que las llevan a sentirse desventuradas, es decir, como si les hubiese tocado, por cosas del azar, transmitir algo malo a sus hijos. En uno de los testimonios se evidencian comparaciones con otros familiares que no heredaron la enfermedad y se percibe esta situación como una mala pasada:

“...uno lo bueno no lo hereda, uno hereda es lo malo, yo decía que uno heredaba lo malo, mire estos dos peladitos hemofilicos y mis sobrinos ninguno salió hemofilico, entonces yo decía que uno nunca hereda lo bueno”. E5

El aprendizaje sobre asumir síntomas, el dolor de sus hijos, el ingreso a las instituciones de salud, la medicación, etc., constituyen un pilar fundamental en el proceso. Aprender, para estas madres, es garantizar para sus hijos que se puedan prevenir las complicaciones conexas con la enfermedad, lo cual las lleva a adquirir unos conocimientos que, a su vez, les permite sobrellevar la enfermedad cuando es necesario:

“... yo me curtí, me curti de verlo echar sangre porque las primeras veces era muy duro, porque a uno al principio le parece horrible lo que le está pasando al hijo, pero de tantas cosas repetitivas uno se va como acostumbrando a verlo así, y uno quiere que no le pase nada a él, uno quiere auxiliarlo, salir volado a conseguir algo con que le remedien a él eso". E3

Las madres, al comprender el hecho de que no se pueden quedar sumidas en los sentimientos de dolor o fatiga, sino que deben retornar hacia sí mismas y valorarse como mujeres, les ayuda a sobreponerse a las adversidades y recobrar la fortaleza.

“... a mí me tocó pelear con la doctora diciéndole que no era amígdalas y oído, jel niño tiene una hemorragia en el cerebro, doctora! porque él tenía un chichón en la frente y no tiene porqué desaparecerse de la noche a la mañana. Y ella me decía, ¿va a saber más usted que es la mamá o yo que soy la doctora? y yo me atreví a decirle: doctora, perdóneme que le diga, pero yo el año rural lo pasé hace 
1794-9831

E-ISSN 2322-7028

Vol. 15 No. 1

Ene - Jun 2018

Cúcuta, Colombia muchos años y no soy doctora, pero yo sé cómo son los hemofilicos..." E3R

Los aprendizajes como pilares fundamentales en el proceso de validar competencias, en el caso de las madres, tienen que ver con aspectos formales e informales. Los primeros apuntan hacia la participación en educación en salud impartida por los profesionales, instituciones de apoyo e instituciones educativas que, en el acceso a los servicios de salud, desarrollan estrategias de acompañamiento para empoderar a la familia mediante la educación. En cuanto a los segundos, se relacionan directamente con la experiencia de cada madre y son aquellos que, desde la cotidianidad, le permiten reconocer las manifestaciones de la enfermedad y acompañar a sus hijos en el cuidado de la misma.

\section{La familia: escenario para el cuidado y la crianza}

La relación con los hijos es reconocida por las participantes como una realidad en la que priman dos componentes: la cercanía, debido al hecho mismo de ser las madres, y la permanencia y dedicación y cuidado adicional que requieren los hijos. Esta permanencia y cercanía hace que se configure un lazo muy fuerte de amor entre madre e hijo que traspasa todas las experiencias; para la madre, los hijos en condición de hemofilia se convierten en el centro de la familia y, desde allí, se construyen las vivencias y relaciones con los demás miembros.

Las madres también dan muestra de la gratificación como acompañantes primordiales de sus hijos; sin embargo, en la experiencia de su proceso vital como portadoras, se presentan ciertas situaciones donde subyacen en los hijos sentimientos hacia la enfermedad que se transmiten a las madres, especialmente en los momentos de crisis. Las madres refieren dos consideraciones que generan en ellas sentimientos de creciente emocionalidad: por una parte, la culpa, la cual es percibida como un asunto infundido desde los hijos más que por una percepción elaborada por ellas mismas; por la otra, se presentan situaciones en las que los hijos emplean la enfermedad para obtener beneficios a través de la manipulación, sobre todo con respecto a hacer y dejar de hacer cosas.

“... los muchachos iban creciendo y no aceptaban lo que les estaba pasando... se iban en represalia contra uno, que nosotros teníamos la culpa, que nosotros por qué no nos habiamos hecho operar antes de casarnos, sabiendo que nosotros sabíamos que en la casa había un hemofilico”. E3

Es precisamente por la forma en que las madres se relacionan con los hijos y por los sentimientos acentuados en el plano de la experiencia como portadoras que éstos pueden ser utilizados para satisfacer sus deseos y censurar sus actuaciones frente a ellos, haciéndolas sentir culpables. Al respecto una madre expresa lo siguiente:

“...él me hace sentir culpable, siempre cuando a él le conviene o quiere lograr algo, siempre me dice que yo esto, que yo lo otro, es que tú no puedes hacer esto porque yo tampoco lo puedo hacer...” E17

Con relación al cuidado, un componente esencial que resaltan las madres es la sensación de soledad. Expresan agradecimiento hacia los familiares cercanos que en ocasiones las acompañan, pero también sienten desasosiego y apatía en sus parejas, quienes, en la mayoría de los casos, no se responsabilizaron de la situación, desligándose de la enfermedad de sus hijos.

Parte de las dificultades para recibir apoyo en el cuidado, en especial por parte de la pareja, se refieren al hecho de hacerlas sentir culpables por ser ellas quienes trasmitieron la enfermedad a los hijos, asunto que se convierte en una de las principales fuentes de conflicto:

“... mi marido se fue de la casa, que porque por el descuido mío el niño se cayó y que porque yo le transmití la enfermedad" E14

Pero el escaso apoyo con respecto al cuidado por parte de la pareja no se limita a no colaborar con el cuidado de los hijos, sino a la ausencia de otras ayudas relacionadas con la necesidad de llevar el sustento económico o saber manejar las crisis de forma adecuada y no dejarlas solas. En el siguiente testimonio se recoge claramente esta situación:

“... en el cuidado como tal a mí me tocó sola, los hombres son muy ofuscados, los hombres quieren que lleguen y los atiendan ahí mismo, como él no sabía cómo era de verdad, a él le tocaba verlos llorar en la casa, pero no sabía lo que era una espera en un hospital, él frente a ellos fue muy aislado, frente a la enfermedad [...] yo prefiero que tenga trabajo y yo soluciono el problema de ellos de salud". E16

Es evidente que la familia es el entorno donde la enfermedad se presenta y también donde se puede solventar; son muchas las percepciones que envuelven 
a las participantes al respecto: fuerza, agradecimiento, indiferencia y, en ocasiones, rupturas, en especial con la pareja. Sin embargo, en su posición como madres portadoras, prima la sensación de ser fuente prioritaria de apoyo y de enlace entre sus hijos y los demás, dentro y fuera del núcleo familiar.

\section{Las mamás y los servicios de salud: entre el apoyo y la lucha}

El apoyo de los profesionales de la salud resulta ser fundamental en el acompañamiento de los hijos. No obstante, en cuanto a la experiencia como portadoras se refiere, puede percibirse como un obstáculo. En ocasiones la información que les brindan se asume como amonestación o censura y la extrapolan a otros familiares, quebrantando las relaciones con los mismos, quienes a partir de éstos forman sus propias consideraciones, adoptando una posición más de juzgamiento que de comprensión.

“...todos los médicos le dicen a uno en las citas: que yo soy la portadora ;muy bien mija que se hizo operar, muy bien hecho! delante del niño le dicen a uno jmuy bien para que va a traer más niños a sufrir!... "E13

Las madres hablan de un saber específico respecto a la forma en que se da la trasmisión de la enfermedad, proporcionado especialmente por los profesionales que las acompañan en los servicios de salud o las redes de apoyo. Al respecto, llama la atención en las madres la necesidad manifiesta de poder acceder a exámenes de laboratorio para tomar decisiones en el momento de concebir a sus hijos; además, porque en algunos casos ellas perciben algunas manifestaciones de la enfermedad que no son manejadas, referidas principalmente a periodos menstruales abundantes, molestias articulares y dificultades en el manejo de la salud bucal.

“... a mí nunca me han hecho pruebas, claro que pues al él ser hemofilico fue porque yo le transmiti ese gen, pero sería muy bueno que a las mamás también nos hicieran pruebas, pero no, yo me di cuenta cuando al niño lo diagnosticaron de que yo también era portadora, al tenerlo a él hemofilico es porque yo le porté la hemofilia...” E1

En tal sentido, una de las participantes manifiesta tener la información pero no comprende bien como es el proceso de trasmisión de la enfermedad -que la madre es portadora y la transmite a sus hijos, y es el hijo hombre quien la padece-:

“... a mí nunca me han hecho exámenes, me explican que al ser alguien portador es porque la mamá es portadora y la mamá de la mamá es portadora, porque la hemofilia la tiene el hombre, aunque yo casi no entiendo... "E9

En casi todos los casos, las madres solicitan ser

\section{tenidas en cuenta en el proceso asistencial, tal vez porque este reconocimiento puede hacer más fácil convivir con la enfermedad de sus hijos y en ellas mismas resolver situaciones de salud que muchas veces no se dan a conocer, tales como la propensión a moretones y hemorragias posteriores a intervenciones quirúrgicas o traumatismos. \\ Las participantes enfatizan en las diferencias que sienten entre ellas y sus hijos y lo beneficioso que sería si las vincularan en el proceso de manera más inclusiva, más humana y, sobre todo, que el acompañamiento no se limite solamente al cuidado de los hijos, sino también a recibir un soporte educativo y profiláctico, enfocado en ellas por parte de los servicios de salud: \\ “...siempre piensan en el niño que tiene hemofilia, no piensan en la mamá, sólo me han dicho usted es portadora, pero nunca me preguntan si me han hecho exámenes, nosotras es como si no estuviéramos en eso, en ese entorno no se interesan en la mamá”. E13 \\ Teniendo en cuenta que el trabajo permitió identificar los discursos y prácticas que se presentan en la relación con las instituciones de salud, cobra sentido pensar en la posibilidad de que las madres sean tenidas en cuenta en el momento de acceder a estos servicios, ya que, aunque desde la educación se ha hecho un trabajo valioso y sirve como contención y soporte para ellas, aún hay restricciones en los modelos de atención en salud, puesto que siguen estando enfocados en los pacientes hombres en condición de hemofilia y para ellas las opciones son limitadas. \\ En todos los casos las madres expresan las variadas peripecias que han tenido que emplear para acceder a los servicios de salud. Concuerdan en que es un deber de los profesionales de la salud proporcionarles herramientas y recomendaciones adecuadas para favorecer una forma de crianza más libre, con menos restricciones y con una comunicación más abierta, en el sentido de lo humano, hacia ellas y sus familias.}


ISSN-PRINT

1794-9831

E-ISSN 2322-7028

Vol. 15 No. 1

Ene - Jun 2018

Cúcuta, Colombia hijos. La llegada de un hijo es una nueva forma de ser y estar en el mundo, pero, cuando ocurre un suceso trascendental como la presencia de una enfermedad crónica como lo es la hemofilia, el panorama es diferente: es necesario sobrellevar la carga de la enfermedad y luchar porque, para su bienestar, es casi obligatorio que todo gire en torno a los hijos.

Esto es lo que expresan las madres en sus experiencias; para ellas portar la enfermedad no es un asunto de inminencia física o bilógica, la enfermedad es como algo que se encarna, que se adhiere a la piel, al corazón y con lo cual se camina y, paradójicamente, es algo que se transforma en valor para afrontar la vida.

Uno de los momentos más trascendentales en su vida que refieren las participantes es la infancia de sus hijos, un período marcado por la intensa energía, la curiosidad y los cambios emocionales. Con la manifestación y diagnóstico de la enfermedad, este proceso se hace más laborioso y se da paso a un deber que no puede dejar de ser cumplido ni ser transferido a cualquier persona, lo que se traduce en dedicación y cuidado absoluto de los hijos. Una de las participantes manifiesta cómo la llegada de su hijo en condición de hemofilia le implicó dedicarse a él tiempo completo y sentir que ella misma pasaba a un segundo plano:

“... a uno le cambian mucho las cosas, porque las 24 ahora son dedicadas a él, uno se olvida de uno mismo, uno ya no existe, uno ya no saca tiempo para arreglarse, uno no saca tiempo para salir, los hijos lo absorben a uno, la vida no es igual". E13

Se puede apreciar cómo las mamás, en algunos momentos, sienten vacíos en su interior; su vida puede resultar monótona al realizar actividades regulares y rutinarias en torno al cuidado del hijo y dejar de hacer cosas en función de ellas.

Otra madre, en su testimonio, refiere que para sentir que sus hijos están bien es fundamental acompañarlos en los momentos de crisis:

“... por la hemofilia he dejado de hacer muchas cosas. Entonces, no puedo trabajar, ellos siempre tienen citas $y$ esto y lo otro y están hospitalizados, entonces no se puede". E11

Para las madres hay varios aspectos que hacen sentir los cambios profundos en su vida a raíz de la enfermedad de sus hijos: las hospitalizaciones, los trámites en las instituciones de salud, el manejo del dolor y las complicaciones repentinas, fenómenos que se van dando en el tiempo y que, a pesar de que las condiciones mejoren, siempre están presentes. Estos cambios se transfieren a todas sus relaciones -de pareja, familia, amistades y demás-, generando un sentimiento general de soledad acrecentado por el amor a sus hijos.

“... a partir del diagnóstico, me descuidé yo mucho, porque todo el tiempo giraba en torno a él, entonces la vida como pareja también se acaba porque eso le genera a uno como una tristeza, o sea, pues como que uno piensa que ya se me cerraron todas las puertas...” E17

En las madres entrevistadas se hace recurrente la necesidad de aplazamiento en el campo profesional, no poder trabajar o culminar los estudios para dedicarse a los cuidados especiales que requieren los hijos y que ameritan gran dedicación, esfuerzo y sacrificios. Los cambios no siempre son negativos, puesto que ellas se dan cuenta que la lucha y la dedicación tienen una retribución fundamental: el hecho de tener a sus hijos con vida y, sobre todo, disfrutarlos en gran medida a su lado.

“... yo creo que mi vida cambió, vale la pena sacrificarse uno por ellos como dice el dicho, hay personas que son muy relajadas, que no se preocupan, que todo lo pueden hacer los médicos y no, hay cosas que uno puede hacer... " E9

Las mamás en algunas ocasiones deben dejar pasar oportunidades en todos los ámbitos, precisamente por la dedicación que conlleva la crianza de un hijo con hemofilia, aunque es notorio que todas tienen algo que pensar y hacer sobre su realización personal, la cual queda aplazada.

\section{Darse cuenta}

Entendida como la conciencia que asumen las madres sobre la enfermedad durante todo el proceso vital, desde las experiencias del padecimiento en el pasado, por antecedentes de familiares con la enfermedad, esperar la enfermedad con la llegada de los hijos, el momento del diagnóstico, sea porque reconocen la enfermedad o porque es la primera vez que se presenta un diagnóstico de hemofilia en la familia, la situación las lleva a plantearse el proceso de saludenfermedad-cuidado de otra manera. Esta categoría emergente se convierte en un proceso transversal a todos los significados de las madres portadoras de hemofilia, es decir, se da en todos los casos y tiene 
que ver con la comprensión que hace cada una de su existencia como portadora, como madre portadora y como acompañante de su hijo en esta condición.

Es común a todas las participantes una conciencia de algo diferente en su vida que trae consigo diversas expresiones en sus vivencias, tales como sufrimiento, vulnerabilidad con respecto a las oportunidades, cambios en las expectativas frente a las relaciones que se construyen con la pareja, los demás familiares y con los servicios de salud, así como en la forma de recibir y asumir la enfermedad. En medio de estas vivencias también se dan transformaciones que les permiten resignificar la experiencia a través del amor de madre y el propio, abriendo posibilidades para la vida en espacios de lucha y regocijo.

\section{Discusión}

Vivir con hemofilia es un suceso que se constituye estructuralmente y cobra sentido frente a la saludenfermedad-cuidado. Como madres, se espera que los hijos nazcan sanos, situación que se ve reflejada en los constructos socioculturales a lo largo de sus vidas, que se expresa como una polaridad manifiesta de tipo físico, psicológico y espiritual y que, principalmente, hace ver las diferencias como algo no aceptable o difícil de sobrellevar.

En el caso específico de la salud, esta polaridad se evidencia en la posición de considerar a las personas como sanas o enfermas. Al respecto, Berlinguer (19) plantea que la enfermedad es socialmente encausada como una desviación más que como diversidad $\mathrm{y}$, habitualmente, se marca la diferencia como anormalidad o inferioridad.

Un aspecto relevante que favorece este análisis es la comprensión de las movilizaciones que hacen las participantes en cuanto al seguimiento del rol como madres al interior y fuera de la familia. La condición de hemofilia como enfermedad crónica y heredada las sitúa en una posición que ellas asumen y ante la cual no están dispuestas a renunciar, puesto que son las cuidadoras primordiales en el proceso de saludenfermedad y en la vida de sus hijos.

En este aspecto, las teorizaciones (20) desde la perspectiva de género ayudan a comprender el papel socialmente asignado a la madre, el cual se enriquece con las voces de las participantes que refieren este proceso como una construcción personal e íntima que no se da por separado sino como un asunto recíproco en el que se imbrica lo que se asume y lo que se asigna: lo primero se refiere a un constructo personal y lo segundo, a las atribuciones que se dan socialmente.

Para comprender estas consideraciones se tienen en cuenta los planteamientos de Berger y Luckman (21), quienes afirman que los roles entendidos como una tipificación aparecen en el contexto del cúmulo de conocimientos objetivados de las personas y son reflejados en su comportamiento, es decir, son un componente esencial para la construcción social y, al interiorizar los roles, ese mismo mundo se recrea subjetivamente en las personas.

En el camino de reconocerse como portadoras de hemofilia, las madres manifiestan momentos de gran soledad debido a la adquisición del rol de cuidadoras primordiales y la representación social de asumir, como mujeres, el cuidado y la crianza de manera casi que exclusiva, conllevando, en ocasiones, a que se resguarden y protejan a sí mismas de los juicios que son trasferidos en las relaciones con los otros, favoreciendo o dificultando la dinámica al interior de la familia.

De igual forma, es común encontrar ciertas categorizaciones de tipo social en cuanto al género, que están cargadas con cierta significación. En el caso de las participantes, es muy frecuente encontrar la frase "eso nos toca", pero, más allá de los constructos sociales que envuelven está asunción de roles, se aprecia que este rol en las participantes toma fuerza por el hecho trascendental de ser madre.

En este sentido, Touraine (22) plantea que el hecho de que las mujeres creen seres humanos les aporta una relación más directa con éstos porque "la mujer desborda los roles sociales gracias a su experiencia de la vida que se gesta en su vientre y que no puede reducirse a un rol institucional. Su función reproductora es lo que introduce, en el caso de la mujer, un desequilibrio con respecto a sus roles sociales".

Así mismo, Lagarde (23) insiste en que el cuidado está en el centro de las contradicciones de género entre mujeres y hombres y, en la sociedad, en la organización antagónica que existe entre sus espacios. El cuidado, como deber de género, es uno 
ISSN-PRINT

1794-9831

E-ISSN 2322-7028

Vol. 15 No. 1

Ene - Jun 2018

Cúcuta, Colombia de los mayores obstáculos en el camino a la igualdad, por su inequidad. En este sentido, es común encontrar que hay ciertas tipificaciones de tipo social en cuanto al género que están cargadas con cierta significación. En el caso de las participantes, es muy frecuente la tipificación de "eso nos toca", pero, más allá de los constructos sociales que envuelven está adjudicación de roles, se aprecia que este rol toma fuerza en ellas por la razón primordial de ser madres.

Otro aspecto fundamental en el cuidado es la relación que las madres portadoras de hemofilia construyen con los servicios de salud -que traspasa el plano de la institucionalidad-, complementando la forma en que se asume la enfermedad, se vive con ella y, en esa medida, se presentan cooperación y dificultades.

Para comprender mejor este aspecto se trae a colación a Menéndez (24), quien plantea que, en todos los contextos, las instituciones asistenciales, de curación, de protección o de control, estructuran una racionalidad que no es exclusivamente técnica ni científica, sino también sociocultural. En esa medida, las prácticas aparecen saturadas de contenidos sociales y culturales que se estructuran no sólo a partir del saber médico sino también en las relaciones que se establecen con los sujetos enfermos y las comunidades y agrega que los factores económicopolíticos son determinantes en la constitución de las formas de estratificación social dominantes.

Desde esta perspectiva se plasman algunos aspectos que permiten entender las brechas que se generan en la relación con los servicios de salud, pero en la medida en que se tengan en cuenta los significados y las experiencias subjetivas en torno a la salud, se pueden favorecer modelos de atención más articulados y con posibilidades para la acción, más relacionadas con la vida de las personas y con el entorno en que se desenvuelven.

Al respecto, Tejada y Mercado (25) plantean que el padecimiento crónico es una condición que perturba de manera desproporcionada la vida de los enfermos y sus familias. Por este motivo, ambos actores viven e interpretan la enfermedad y su atención desde su propio mundo, sus necesidades y temores. En este proceso pueden coincidir en algunas dimensiones pero no necesariamente en todas: las madres plantean, por ejemplo, que mientras más avanzan los tratamientos médicos, paradójicamente es cuando menos interés hay por las condiciones de vida de quienes padecen la enfermedad y sus familiares.

Es un desafío comprender la forma en que las madres portadoras de hemofilia dan sentido a su existencia, pues más que explicaciones o vivencias fáciles o aprendidas, sus relatos describen vicisitudes, luchas y logros que las llevan a convertirse en las protagonistas de su vida desde una mirada potencial y no de víctimas.

Al respecto, la psicología humanista declara que hay una fuerza en todos los seres humanos que lleva a buscar los medios para la realización personal y para encontrar el sentido de la vida en el seno de las potencialidades del ser; la autorrealización exige una lucha de por vida y superación de dificultades mediante la integración de diferentes propósitos, también habla de diversos estímulos que sirven como motivación y que se pueden dar bajo tensión interna constante (26).

Es indudable que las madres tienen una motivación fundamental en su vida, sus hijos, y en esa medida es que transforman sus experiencias dolorosas y se convierten en la fuerza fundamental para ambos. Para entender mejor esta consideración, se plantea la perspectiva de Frankl (27), quien afirma que "como el sufrimiento es un aspecto que no puede apartarse de la vida, al hombre se le puede arrebatar todo salvo una cosa: la última de las libertades humanas -la elección de la actitud personal ante un conjunto de circunstancias- para decidir su propio camino, y esa libertad espiritual es la que hace que la vida tenga sentido y propósito".

Las madres portadoras de hemofilia, estando inmersas en un grupo social, en una familia y cada una con sus diferencias, dejan ver claramente esta capacidad de elección que tienen frente a su proceso. La enfermedad también propicia el sentido de vida y ellas aprenden a caminar con algo de lo que no se pueden desprender.

Teniendo en cuenta que se identificaron los discursos y prácticas que se presentan en la relación con las instituciones de apoyo y de salud, cobra sentido pensar en la posibilidad de que las madres sean tenidas en cuenta en el momento de acceder a los servicios de salud, ya que, aunque desde la educación se ha hecho un trabajo valioso y sirve como contención y soporte para ellas, aún hay restricciones en los modelos de atención en salud, es decir, los servicios siguen 
estando enfocados en los hombres en condición de hemofilia y para ellas las opciones son limitadas.

Es por esto que se plantea la importancia de que las madres tengan otros espacios como portadoras -que traspase el plano de cuidadoras- que involucre exámenes de detección y acompañamiento de corte interdisciplinario. Esto puede favorecer las decisiones en cuanto a la concepción y el acompañamiento a sus hijos, además de resolver cuestiones de su salud y mejorar su bienestar desde el ámbito clínico y asistencial.

La Federación Mundial de Hemofilia (28) plantea la importancia de detectar las portadoras e incluirlas en los procesos de atención en salud. Existen dos tipos de portadoras: las obligadas, que necesariamente tienen el cromosoma $\mathrm{X}$ afectado, $\mathrm{y}$ las probables.

"Son portadoras obligadas: todas las hijas de un hombre con hemofilia, madres de un hijo con hemofilia y por lo menos otro familiar con hemofilia (hermano, abuelo materno, tío, sobrino o primo), madres de un hijo con hemofilia y una familiar que sea portadora del gen de la enfermedad (madre, hermana, abuela materna, tía, sobrina o prima), madres de dos o más hijos con hemofilia. Son portadoras probables: todas las hijas de una portadora, madres de un hijo con hemofilia que no tienen otros familiares con la enfermedad o portadoras de ella, hermanas, madres, abuelas maternas, tías, sobrinas y primas de portadoras" (28).

Para ampliar el panorama alrededor de las consideraciones clínicas y la sintomatología que se puede presentar en las madres, la guía de la Federación Mundial de Hemofilia (29) sobre portadoras y mujeres en esta condición, plantea, al respecto, que aproximadamente un tercio de las portadoras tienen niveles de factor de coagulación en la sangre menores de lo normal y podrían presentar hemorragias anormales. En la mayoría de los casos, las portadoras experimentan síntomas similares a los vistos en hombres con hemofilia leve, así como algunos otros específicos de las mujeres, tales como periodos menstruales abundantes o prolongados.

Las portadoras sintomáticas pueden presentar moretones más fácilmente, hemorragias prolongadas después de cirugías y traumatismos, con frecuencia tienen periodos menstruales más abundantes y prolongados (menorragia) y más probabilidades de necesitar un suplemento de hierro o de ser sometidas a una histerectomía; de igual forma, tienen más probabilidades de presentar hemorragia posparto después del alumbramiento (29).

La guía de la Federación Mundial de Hemofilia también retoma aspectos psicosociales y de la calidad de vida y, en resumen, plantea lo siguiente:

"Muchos centros de tratamiento de hemofilia pueden ofrecer a las portadoras asesoría experta y sensible. Sus profesionales pueden proporcionar información y apoyo para lidiar con los complejos sentimientos y habilitar a las mujeres para que se hagan cargo de su trastorno y aboguen por su tratamiento adecuado. El establecimiento - a través del centro de tratamiento de hemofilia u organización de pacientes locales- de una red de apoyo formada por otras mujeres que enfrentan los mismos problemas puede resultar sumamente reconfortante" (29).

Teniendo en cuenta estas consideraciones, las apreciaciones de las participantes y la creciente preocupación en el país por el impacto que tiene el tratamiento de la enfermedad en el Sistema General de Seguridad Social en Salud. El ministro de salud, Alejandro Gaviria, afirmó en Abril 29 del 2014 que la hemofilia es la enfermedad que mayor carga financiera le genera al sistema de salud colombiano anualmente, dado que las 2.200 personas que la padecen requieren de una inversión que supera los $\$ 400.000$ millones de pesos (30).

Pensar en opciones de inclusión en el ámbito social y de la salud para las madres portadoras puede ser el punto de partida para conciliar un vínculo personasociedad-estado, promoviendo cambios en el sistema mediante una dimensión mucho mayor a través del reconocimiento de las dificultades de la población y generando estrategias conjuntas que reduzcan los costos y consideren al ser humano en la totalidad de sus aspectos. Esto requiere de una mirada propia de la salud colectiva que fomente acciones a favor de procesos salud-enfermedad-cuidado de manera interdisciplinaria, sobre todo para replantear las nociones y construir una salud centrada en la vida, donde lo social no se conciba como algo secundario, externo o alejado de lo biológico.

\section{Conclusiones}

- La salud colectiva es la posibilidad de tener un acercamiento más comprensivo al proceso salud- 
ISSN-PRINT

1794-9831

E-ISSN 2322-7028

Vol. 15 No. 1

Ene - Jun 2018

Cúcuta, Colombia enfermedad-atención de los grupos humanos, tomando en cuenta las condiciones económicas, sociales y culturales en las que éstos se inscriben. En este sentido, la pregunta por los significados que construyen las madres acerca de ser portadoras de hemofilia, develó el tejido de lo individual y lo colectivo como un asunto que trasciende el plano biológico de la enfermedad y da cuenta de la construcción social en la que confluyen prácticas, saberes, imaginarios y sentimientos.

- Uno de los aspectos más relevantes desde esta perspectiva, que se plasmó en el proceso, consiste en que a través del método cualitativo y el enfoque del interaccionismo simbólico se abrieron las puertas para que se potenciara la forma de entender la experiencia de las madres como portadoras de la enfermedad. Las entrevistas permitieron establecer un vínculo más profundo con las participantes hasta llegar a los significados $\mathrm{y}$, desde sus particularidades, se lograron evidenciar las situaciones de tipo social que son comunes a una población y que pueden abrir las posibilidades a otras alternativas de intervención.

- Las madres reconocen los avances en la oferta de los servicios de salud y el tratamiento de la enfermedad; sin embargo, en ocasiones se pierde de vista la realidad de los pacientes y sus familiares. Ellas plantean la importancia de tener otros espacios más allá del plano de cuidadoras, en donde puedan acceder a exámenes de detección y acompañamiento interdisciplinario. Esto puede favorecer las decisiones en cuanto a la concepción y el acompañamiento a sus hijos y, en parte, resolver cuestiones de su salud, mejorando su bienestar desde el ámbito clínico y asistencial.

\section{Conflicto de Intereses}

La autora declara no tener ningún conflicto de intereses.

\section{Referencias Bibliográficas}

1. Gómez S, Mantilla F, Beltrán J, Jaloma R. Diagnóstico molecular en pacientes y portadoras de hemofilia A y B. Gaceta Médica de México. [Internet]. 2005. [consultado 24 de octubre de 2017]; 14(1):69-71. Disponible en: http://www.scielo.org.mx/scielo.php?script=sci_arttext\&pid=S0016-38132005000100012

2. World Federation of Hemophilia (WFH). Guidelines for the Treatment of Hemophilia: General care and management of hemophilia. 2nd ed. [Internet]. Montreal, Canadá: Blackwell Publishing Ltd.; 2016 [consultado 3 de junio de 2016] 21-23. Disponible en: http://www1.wfh.org/publication/files/pdf-1472.pdf

3. Federación Mundial de Hemofilia (FMH). Perspectivas de las portadoras de hemofilia. Edición revisada. Tratamiento de la Hemofilia. [Internet]. 2004 junio [consultado 10 de mayo de 2016]; (8): 1-6. Disponible en: http://www1.wfh.org/publication/files/pdf-1513.pdf

4. Liga Antioqueña de Hemofílicos. Programas de hemofilia en Colombia. Registros documentales: Liga Antioqueña de Hemofílicos. Medellín: Liga Antioqueña de Hemofílicos; 2009.

5. Federación Mundial de Hemofilia (FMH). Asesoría Genética en hemofilia. Tratamiento de la hemofilia. [Internet]. 2015 Julio [consultado 7 de junio de 2016]; (25): 1-18. Disponible en: http://www1.wfh. org/publications/files/pdf-1161.pdf

6. Bermeo SM, Silva CT, Fonseca DY, Restrepo CM. Hemofilia: diagnóstico molecular y alternativas de tratamiento. Colomb Med [Internet]. 2007 [consultado 4 de junio de 2016]; 38(3): 308-315. Disponible en: http://www.bioline.org.br/pdf?rc07054

7. Federación Mundial de Hemofilia (FMH). Opciones de tratamiento para la atención de la Hemofilia en países en vías de desarrollo, [Internet]. 2005 diciembre [consultado 31 de octubre de 2017] Disponible en: http://www1.wfh.org/publication/files/pdf-1185.pdf

8. Neira Rosas JA. Ciencias Sociales y salud pre-textos para el debate. Rev. Austral Ciencias Sociales 2001; 5:127-138.

9. Morse JM. Asuntos críticos en los métodos de investigación cualitativa. Medellín: Universidad de Antioquia; 2003. 193, 185-214.

10. Galeano Marín ME. Registro y sistematización de información cualitativa. [Internet]. 2001 [consultado 
5 de mayo de 2016]. Disponible en: https://www.academia.edu/6608261/Registro_y_sistematizacion_ de_informacion_cualitativa

11. Sandoval Casilimas CA. Investigación cualitativa. Programa de especialización en teoría métodos y técnicas de investigación social. Bogotá: Instituto Colombiano para el Fomento de la Educación Superior (ICFES); 1996.

12. Goetz J, LeCompte MD. Etnografía y diseño cualitativo en investigación educativa. Ediciones Morata: Madrid; 1988. Págs. 128-129.

13. Galeano ME. Diseño de Proyectos en la Investigación Cualitativa. Medellín: Universidad EAFIT; 2003. Pág 35-38.

14. Strauss A, Corbin J. Codificación axial. Bases de la investigación cualitativa. Técnicas y procedimientos para desarrollar la teoría fundamentada Medellín: Universidad de Antioquia; 2002.

15. Menéndez E. El punto de vista del actor. Homogeneidad, diferencia e historicidad. [Internet] Relaciones 1997; XVIII (69:237-270) [Consultado octubre 25 de 2017] Disponible en: http://www.scielo.org.co/ scielo.php?script $=$ sci_nlinks\&ref $=000123 \&$ pid $=$ S0120-386X200700010000600015\&lng=en

16. Consejo de Organizaciones Internacionales de las Ciencias Médicas - Organización Mundial de la Salud. Pautas éticas internacionales para la investigación biomédica en seres humanos. Edición en lengua española. [Internet]. Ginebra; 2002 [consultado julio de 2016]. Disponible en: http://www. ub.edu/rceue/archivos/Pautas_Eticas_Internac.pdf

17. República de Colombia. Ministerio de Salud. Resolución No 008430 de 1993, octubre 4, por la cual se establecen las normas científicas, técnicas y administrativas para la investigación en salud. [Internet]. Bogotá: Ministerio de Salud; 1993 [consultado 21 de mayo de 2016]. Disponible en: https://www. minsalud.gov.co/sites/rid/Lists/BibliotecaDigital/RIDE/DE/DIJ/RESOLUCION-8430-DE-1993.PDF

18. Castro R. La vida en la adversidad: el significado de la salud y la reproducción en la pobreza. México; 2000.

19. Berlinguer G. La enfermedad: sufrimiento, diferencia, peligro, señal, estimulo. Buenos Aires: Lugar Editorial; 2007.

20. Montes S. Las Mujeres y la Salud. Compilado. Programa interdisciplinario de estudios de la mujer. México. 1995.

21. Berger PL, Luckman T. La construcción social de la realidad. Buenos Aires: Amorrortu editores; 2003.

22. Touraine A. El mundo de las mujeres. Barcelona: Paidós; 2007.

23. Lagarde M. Mujeres cuidadoras: entre la obligación y la satisfacción. En: Comunidad Europea, Fondo Social Europeo. Congreso Internacional Sare: Cuidar cuesta: costes y beneficios del cuidado. [Internet]. Vitoria Gasteiz: Emakunde, Instituto Vasco de la Mujer; Septiembre 2004. [consultado 29 de septiembre de 2017]: p. 155-160. Disponible en: http://www.emakunde.euskadi.eus/contenidos/ informacion/pub_jornadas/es_emakunde/adjuntos/sare2003_es.pdf

24. Menéndez E. La enfermedad y la Curación ¿Qué es medicina tradicional? Alteridades [Internet]. 1994 [consultado 28 de junio de 2016]; 4(7)71-83. Disponible en: http://biblioteca.ues.edu.sv/ revistas/10800276-8.pdf

25. Tejada LM, Mercado F. La atención médica a los enfermos crónicos: contribuciones de una evaluación cualitativa. México: Plaza y Valdez Editores; 2013.

26. Quimann H. Psicología Humanística: conceptos fundamentales y trasfondo filosófico. Barcelona; Editorial Herder; 1989.

27. Frankl V. El hombre en busca de sentido. Barcelona: Editorial Herder; 1991.

28. World Federation of Hemophilia. Symptomatic carriers of hemophilia. Treatment of Hemophilia. [Internet]. 2008 [consultado 7 de marzo de 2015]; (46):1-12. Disponible en: http://www1.wfh.org/ publication/files/pdf-1202.pdf

29. World Federation of Hemophilia. Carriers and women with hemophilia. [Internet]. WFH; 2012 [consultado 3 de junio de 2016]. Disponible en: http://www.wfh.org/en/abd/carriers/carriers-andwomen-with-hemophilia-en

30. Hemofilia, la enfermedad más costosa para el sistema de salud. El Tiempo. [Internet]. Martes 29 de abril de 2014: Redacción Salud. [consultado 29 de abril de 2016]. Disponible en: http://www.eltiempo. com/archivo/documento/CMS-13901057 